\title{
New perspectives on thermosphere tides: 2. Penetration to the upper thermosphere
}

\author{
Jeffrey M Forbes ${ }^{1 *}$, Xiaoli Zhang ${ }^{1}$ and Sean L Bruinsma ${ }^{2}$
}

\begin{abstract}
In this paper, we present new multi-year and 72-day mean seasonal-latitudinal tidal structures in exospheric temperature derived from joint analysis of CHAMP and GRACE accelerometer measurements. These results include diurnal tides DE3, DE2, D0, and DW2 and semidiurnal tides S0, SE1, SE2, SE3, SW4, and SW6. We also employ Hough mode extensions (HMEs) and the Climatological Model of Thermosphere Tides (CTMT) to ascertain whether the observed structures are consistent with those observed at $110 \mathrm{~km}$ and presented in part 1 of this study. The aggregate sum of all the tidal components is shown to impose considerable longitude and month-to-month variability on the exosphere temperature tidal spectrum.

Please see related article: http://www.earth-planets-space.com/content/66/1/136.
\end{abstract}

Keywords: Tides; Thermosphere; Dynamics; Propagation

\section{Background}

The degree to which the ionosphere-thermosphere (IT) system is linked to lower atmosphere variability is a fascinating discovery of the past decade or so. Much if not all of this coupling occurs via the vertical propagation of waves. In part 1 of this two-part study, we revealed the seasonal-latitudinal dependences of the mean tidal spectrum entering the IT system at $110 \mathrm{~km}$ altitude. This is the tidal spectrum that is likely driving electric fields of tidal origin in the dynamo region (100 to $150 \mathrm{~km}$ ) of Earth's ionosphere, and as such is highly relevant to the interpretation of F-region plasma drifts and the plasma redistributions that they produce. In this context, we further quantified the seasonal-latitudinal structures of the migrating DW1, SW2, and TE3 tidal components (see part 1 for the tidal nomenclature we are employing here), as well as other tidal components that are often quoted in the literature such as DE3 and DE2. In addition, we provided new insights into the seasonal-latitudinal structures of components of the semidiurnal part of the spectrum that have received little or no attention in the literature, such as SE1, SE2, SE3, S0, SW4, and SW6. For all of these waves, one might ask to what degree they are capable of

\footnotetext{
*Correspondence: forbes@colorado.edu

${ }^{1}$ Department of Aerospace Engineering Sciences, University of Colorado, 1111 Engineering Drive, Boulder, CO 80309, USA

Full list of author information is available at the end of the article
}

penetrating into higher regions of the IT (i.e., $400 \mathrm{~km}$ )? It is one purpose of this paper to answer this question.

A related question is 'What is the non-migrating tidal spectrum in the upper thermosphere, and how does it relate to that at $110 \mathrm{~km}$ ?' The answer is not so direct, as the former is not simply related to the latter vis$\grave{a}$-vis vertical propagation. There are other mechanisms that produce non-migrating tides in the IT system. For instance, as shown by Hagan et al. (2009) and to some degree verified by Oberheide et al. (2011a), the nonlinear interaction between DE3 and DW1 produces SE2 and a stationary planetary wave with $s=4$ (SPW4). We might expect, therefore, that additional tidal components are produced by other nonlinear wave-wave interactions. In addition, Jones et al. (2013) demonstrate that the longitudinally varying ionosphere can, through ion drag interactions involving the DW1 (migrating or Sun-synchronous) wind field excited in situ in the thermosphere, produce significant D0 and DW2 tidal components during nonsolar-minimum conditions. The in situ generation of these waves was in fact suggested by Oberheide et al. (2011b) based on CHAMP accelerometer measurements, and observational evidence for an in situ driven D0 is provided by Lieberman et al. (2013). Jones et al. (2013) have shown that additional diurnal and semidiurnal tidal components are generated with this mechanism and with greater efficiency for higher levels of solar activity.

\section{它 Springer}

(c) 2014 Forbes et al:- licensee Springer. This is an Open Access article distributed under the terms of the Creative Commons Attribution License (http://creativecommons.org/licenses/by/4.0), which permits unrestricted use, distribution, and reproduction in any medium, provided the original work is properly credited. 
The purpose of this paper is twofold. First, we analyze CHAMP and GRACE accelerometer data to reveal the full spectrum of non-migrating (i.e., longitude-dependent) diurnal, semidiurnal, and terdiurnal tides in the upper thermosphere and the seasonal-latitudinal structure of each component. A new aspect of this tidal analysis is that we exploit the local time coverage afforded by combining CHAMP and GRACE data together and thus derive tides in terms of 72-day mean values with exosphere temperature being the dependent variable. Previous tidal analyses with CHAMP were performed in terms of perturbation density and consisted of 130-day mean values due to the slow local time precession of the CHAMP orbit. This type of averaging likely suppressed actual amplitudes and significantly smeared month-to-month variability. In the context of tidal theory applicable to the thermosphere, we then attempt to ascertain to what degree the spectral components at $400 \mathrm{~km}$ arise due to vertical propagation from $110 \mathrm{~km}$ and, by extension, what components might be excited in situ within the IT system.

The following section describes how we analyze the CHAMP and GRACE accelerometer data to derive the tidal spectrum as well as the elements of tidal theory employed in our analysis. The subsequent 'Analysis of CHAMP and GRACE data' section presents results for the diurnal, semidiurnal, and terdiurnal components of the observed upper thermosphere tides, including comparisons with TIMED/SABER at $110 \mathrm{~km}$ and discussions of possible origins of the illustrated waves. This is followed by presentations and discussions of the total latitude, longitude, and monthly variability of diurnal and semidiurnal exosphere temperature amplitudes in the 'Results and discussion' section. The 'Conclusions' section includes a brief summary of the results and conclusions.

\section{Methods}

\section{HMEs and the CTMT}

In this paper, we will refer to and utilize Hough mode extensions (HMEs) and the Climatological Model of Thermosphere Tides (CTMT) in the interpretation of results. The concept of HMEs was developed by Lindzen et al. (1977) and Forbes and Hagan (1982) in order to deal with the changes in shape of Hough modes as they encountered dissipation in an atmospheric regime above that of wave forcing. A Hough mode extension represents the global solution (pole-to-pole, 0 - to $400-\mathrm{km}$ altitude) to the linearized dynamical equations of the atmosphere for an oscillation of a given frequency and zonal wavenumber, taking into account dissipative effects (i.e., radiative cooling, eddy and molecular diffusion of heat and momentum) above the forcing region. These perturbation fields are calculated with respect to a background windless atmosphere characterized by a single climatological height profile of temperature.
The HMEs are forced with a conveniently normalized heat source confined to the troposphere and with latitude shape given by the corresponding classical Hough mode. For a given zonal wavenumber and frequency, an HME can be thought of as a latitude versus height table of amplitudes and phases for the velocity, temperature, and density perturbation fields $(u, v, w, T, \rho)$ of the oscillation. The $(u, v, w, T, \rho)$ perturbation fields maintain internally selfconsistent relative amplitude and phase relationships for any given HME. So, if the amplitude and phase of the perturbation wind field are known for a given HME at a single latitude and height, then all the fields $(u, v, w, T, \rho)$ are known for all latitudes and all heights.

The methodology for fitting HMEs to observational data is fully described in Forbes et al. (1994). These authors used HMEs for the migrating semidiurnal tide to simultaneously fit semidiurnal tidal winds and temperatures between 80 and $150 \mathrm{~km}$ and, by reconstruction, arrived at a monthly climatological model of horizontal and vertical winds, temperatures, and densities in this height region. Subsequently, Svoboda et al. (2005) utilized HMEs for migrating and non-migrating diurnal tides to fit UARS tidal winds at $95 \mathrm{~km}$ to similarly arrive at an internally consistent global climatology of tidal temperatures, winds, and densities in the 80 - to $120-\mathrm{km}$ height region.

Figure 1 illustrates the amplitude and phase vertical structures of a few key temperature HMEs at the latitudes of maximum temperature (generally, at the equator for the first symmetric HMEs and near $18^{\circ}$ to $24^{\circ}$ latitude for the first antisymmetric HMEs). In this depiction, all amplitudes are normalized to a value of unity at $100 \mathrm{~km}$, and phases at $100 \mathrm{~km}$ are adjusted to $24 \mathrm{~h}$ for diurnal components and $12 \mathrm{~h}$ for semidiurnal components, for ease of comparison. Although the horizontal shapes at $100 \mathrm{~km}$ are close to the corresponding Hough functions depicted in part 1 , the horizontal shapes tend to broaden with height, and nodes move in latitude. This is because the addition of diffusion renders the tidal equations inseparable.

The top row of Figure 1 shows the amplitudes and phases of the first symmetric and antisymmetric components of D0, DE2, and DE3. As tabulated by Oberheide et al. (2011b), the vertical wavelengths between 90 and $110 \mathrm{~km}$ of the symmetric components are 32, 70, and $42 \mathrm{~km}$, respectively, while those of the corresponding antisymmetric components are 69,35 , and $28 \mathrm{~km}$. The longer vertical wavelength waves penetrate more effectively to higher altitudes than those with shorter vertical wavelengths, since the latter are more susceptible to molecular dissipation than the former. That is, exponential growth with height ceases near the altitude where the time constant for molecular dissipation equals the period of the wave, and this occurs at higher altitudes for larger vertical wavelengths (e.g., Lindzen 1968). So, we 

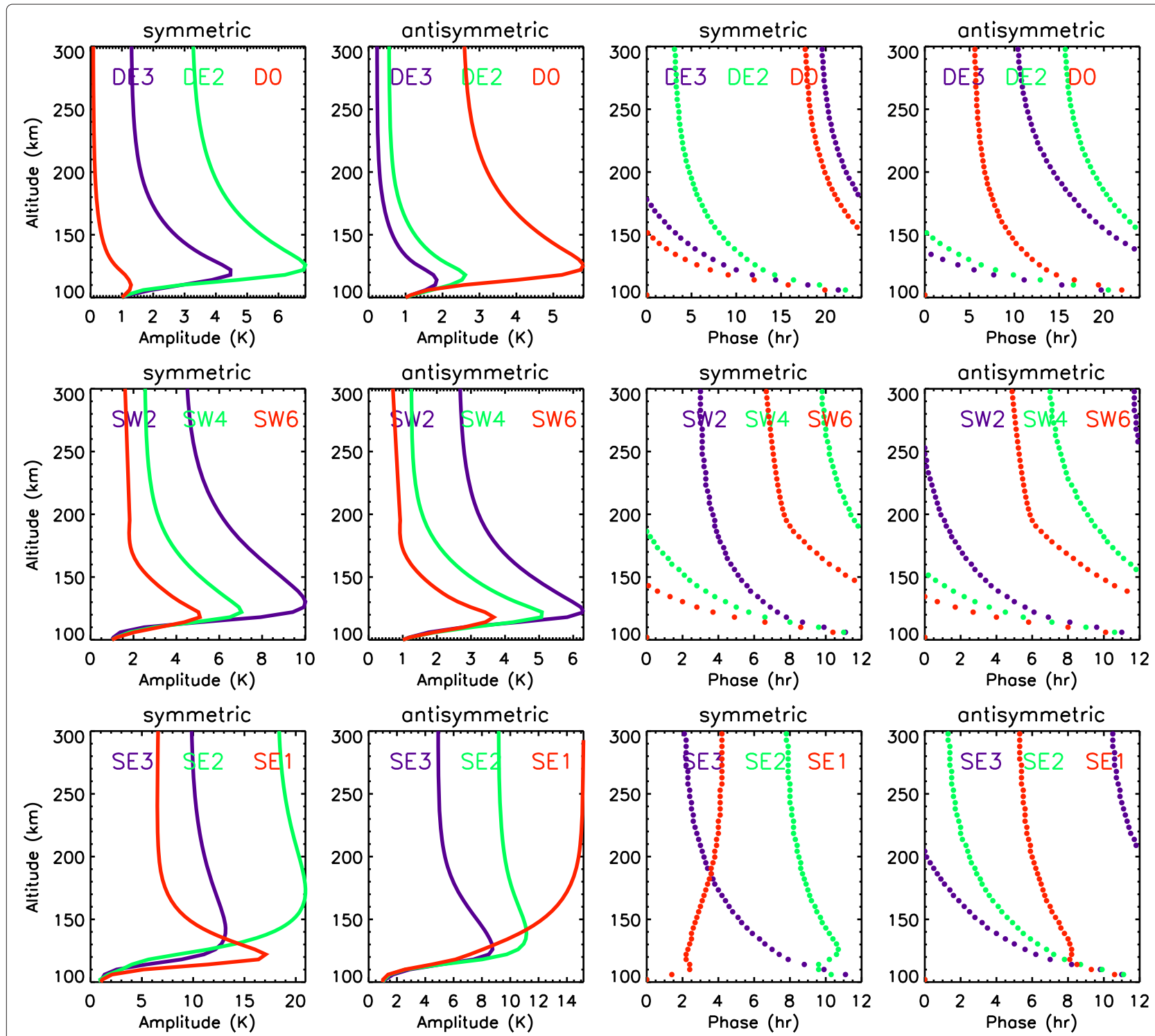

Figure 1 Amplitude and phase vertical structures. HME temperature amplitude and phase vertical structures of the various non-migrating diurnal and semidiurnal tidal components considered in this study.

expect the antisymmetric component of D0 and the symmetric components of DE2 and DE3 to penetrate more efficiently into the upper thermosphere. Also, waves with vertical wavelengths of less than about $30 \mathrm{~km}$ do not efficiently propagate to higher altitudes in the thermosphere. Therefore, we would expect DE3 and to some degree DE2 to take on a symmetric character in the upper thermosphere and for D0 to take on a more asymmetric character; however, factors such as ion drag and zonal-mean winds in the thermosphere can add further complications.

Similar arguments apply to SW1 to SW6 and SE1 to SE3 which have progressively shorter vertical wavelengths. The first symmetric components of DE1, SE1, SE2, and
SE3 all have vertical wavelengths greater than $100 \mathrm{~km}$ below $100 \mathrm{~km}$ altitude and are evanescent in the so-called mesospheric barrier (Geller 1970) between about 80 and $100 \mathrm{~km}$. As noted in part 1, the symmetric components of these waves are small compared to their antisymmetric counterparts around 100 to $110 \mathrm{~km}$. However, as these waves emerge from this barrier, their behaviors change as they encounter enhanced dissipation and thus deviations from classical Hough mode structures occur, sometimes producing apparently anomalous behavior such as the vertical structure of SE3 in the bottom panel of Figure 1. However, since the temperature fields associated with these waves at the base of the thermosphere are predominantly antisymmetric, the behavior of their symmetric 
components becomes inconsequential in the current context. These characteristics of HMEs will serve as a basis for interpreting the results to be presented in the 'Results and discussion' section.

\section{Analysis of CHAMP and GRACE data}

As noted above, a hindrance of using CHAMP data to derive thermosphere tides is the 130-day time period it takes for the CHAMP satellite to sample $24 \mathrm{~h}$ of local time, even when considering sampling along both the ascending and descending parts of the orbit; over 130 days, tidal amplitudes and phases can vary considerably, and the 130-day mean tidal determinations can lead to a significant underestimate of the tidal amplitudes that occurred within the fitting interval. In Forbes et al. (2009), we took advantage of the relative precession rates of the CHAMP and GRACE satellites, and the fact that exosphere temperature is independent of height in the 350- to $550-\mathrm{km}$ height regime, and demonstrated the feasibility of extracting 72-day running means of tidal components in exosphere temperature. In the present paper, we apply this method to the CHAMP and GRACE data sets extending to 2010, with the intent of establishing the full spectrum of non-migrating (i.e., longitude-dependent) diurnal, semidiurnal, and terdiurnal tides that exist at orbital altitudes in Earth's thermosphere. With a 72-day moving window, we are able to delineate tides with much improved resolution and variability compared with those obtainable
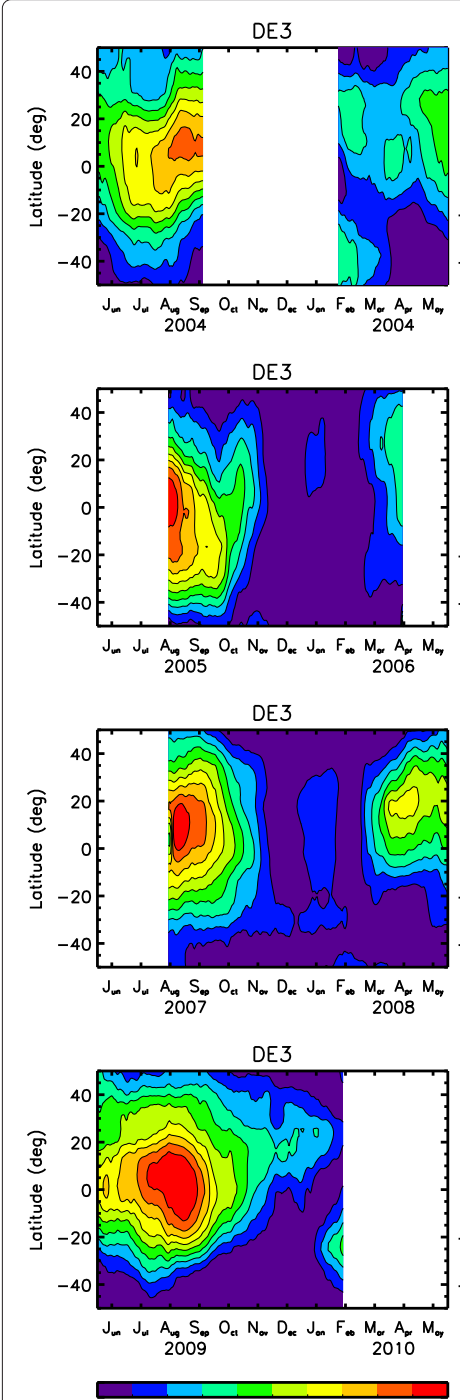

0.81 .21 .62 .02 .42 .83 .23 .64 .0

(K)

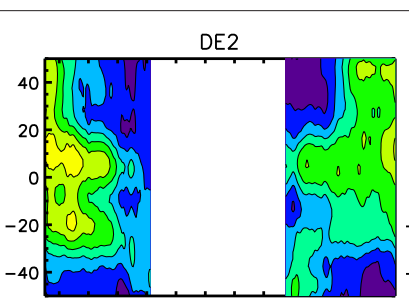

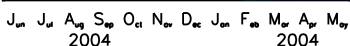
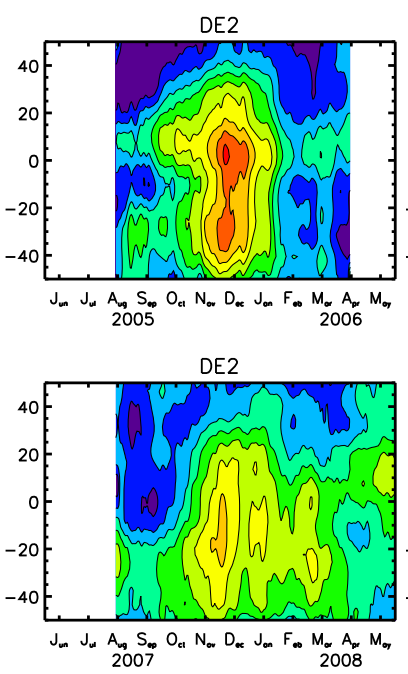

DE2

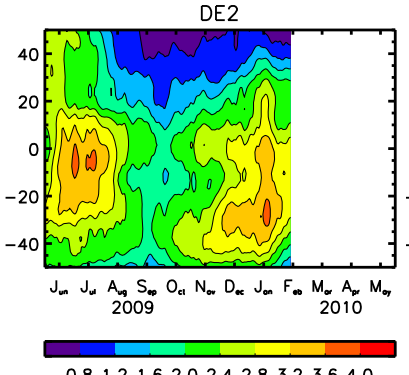

$\begin{array}{llllllllll}0.8 & 1.2 & 1.6 & 2.0 & 2.4 & 2.8 & 3.2 & 3.6 & 4.0\end{array}$

$\mathrm{T}(\mathrm{K})$
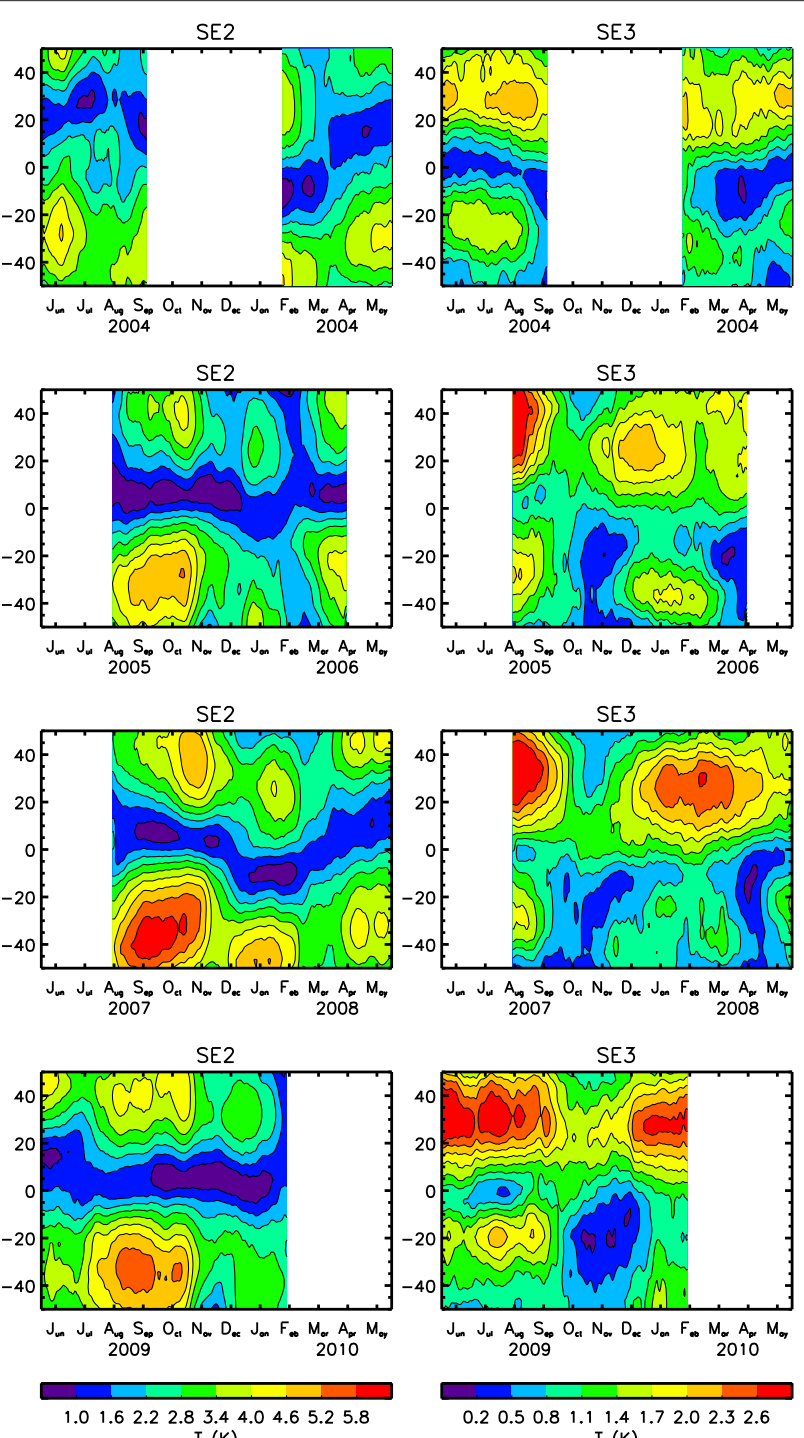

$\mathrm{T}(\mathrm{K})$

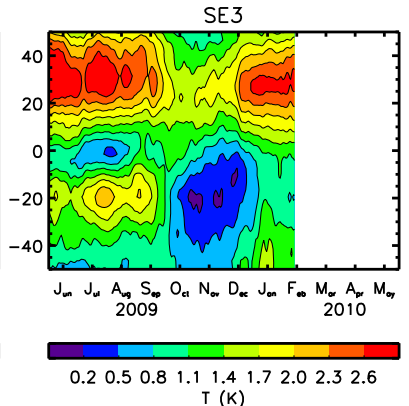

$\mathrm{T}(\mathrm{K})$

Figure 2 Latitude vs. month depictions of tidal amplitudes. Sample plots providing latitude vs. month depictions of tidal amplitudes of exosphere temperature for the four time intervals (top to bottom) considered in this study: DE3, first column; DE2, second column; SE2, third column; SE3, fourth column. 
from CHAMP or GRACE alone. The reader is referred to Forbes et al. (2009) for a detailed description of the method, error analysis, and validation. A brief summary is provided below.

As a first step, the CHAMP and GRACE densities are converted to equivalent exosphere temperatures. CHAMP and GRACE densities are measured along noncircular orbits and at different altitudes within the 300to $550-\mathrm{km}$ height regime. In order to combine these data together for a tidal analysis, the densities are first converted to equivalent exosphere temperatures. After the CHAMP and GRACE densities are inter-calibrated, the conversion to exosphere temperature is done empirically by iterating on the solar flux input into the NRLMSISE-00 empirical model (Picone et al. 2002) until the model and measured density agree. The parametric dependence between exosphere temperature and density makes this possible. Although the derived exosphere temperatures may contain a bias imposed by the model, any bias has a small effect since the analysis is performed on perturbations about the mean temperature. In addition, for the most part, NRLMSISE-00 does not include the non-migrating tides we are seeking to recover in the analysis, except perhaps at high latitudes where convolution between longitude and local time can occur in connection with polar and auroral processes (see, e.g., Forbes et al. 2012; Jones et al. 2013). For this reason, and to conform with the SABER temperature analyses in part 1 , we restrict our analysis to the latitude range between $\pm 50^{\circ}$. Within this latitude range, NRLMSISE-00 does not contain the types of tidal longitude variations that we extract from the CHAMP and GRACE data, precluding the possibility that such variations could arise through the density-temperature conversion process.

As a second step, exosphere temperatures covering $24 \mathrm{~h}$ of local time and $360^{\circ}$ longitude (about 14 to 15 orbits per day) are subjected to two-dimensional Fourier fitting to extract the various non-migrating tidal components. Key to the methodology applied here, the CHAMP and GRACE orbits precess in local time with respect to each other. It turns out that the combined sampling offered by these two satellites on both ascending and descending parts of their orbits results in complete local time sampling during 72-day intervals. Of course, there are periods of time around co-planar events when this type
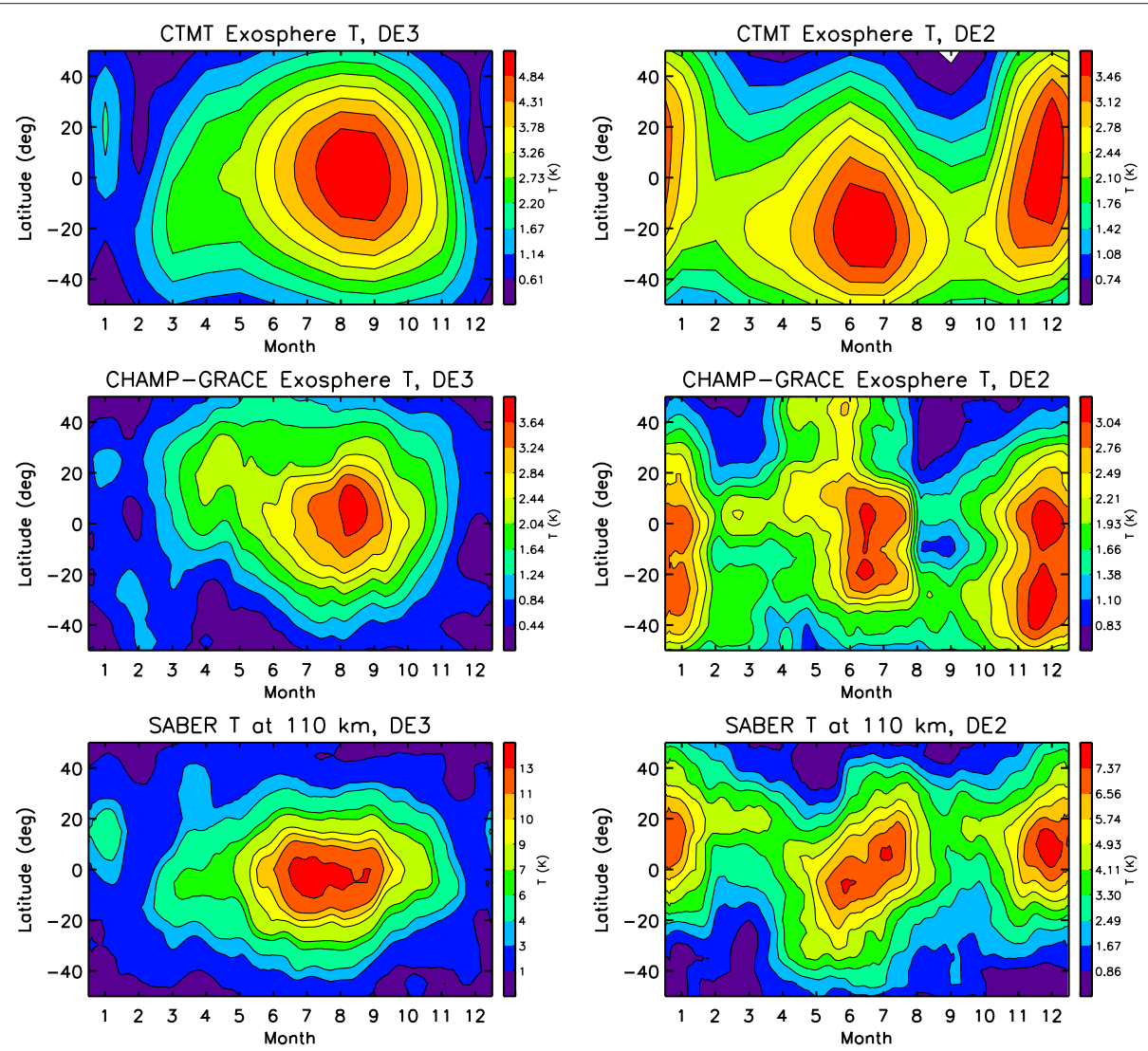

Figure 3 Latitude vs. month depictions of select tidal components. For four-interval vector mean SABER temperatures at $110 \mathrm{~km}$ (bottom), four-interval vector mean CHAMP-GRACE exosphere temperatures (middle), and CTMT exosphere temperatures (top) for DE3 (left) and DE2 (right). 
of sampling is not possible. As it turns out, there are four periods of time during which tidal analyses can be performed to provide daily 72-day running mean determinations of the diurnal, semidiurnal, and terdiurnal tides: 8 February 2004 to 20 September 2004, 15 August 2005 to 17 May 2006, 15 August 2007 to 11 June 2008, and 15 May 2009 to 16 February 2010.

The DE3, DE2, SE2, and SE3 exosphere temperature tidal amplitudes derived by the above methodology during these four intervals are provided in Figure 2. For the most part, there is inter-annual consistency between these patterns, as the 81-day mean F10.7 solar index only varies between about 110 and 70 solar flux units during the whole time period. Finally, in order to provide consistent depictions with the climatological results from SABER in part 1 and CTMT in following sections, these tidal determinations are vector averaged to obtain a single annual climatology. These annual climatologies are depicted in Figures 3, 4, 5, 6 and 7, which are discussed in the following section. For consistency in the following depictions, the SABER results at $110 \mathrm{~km}$ are averaged over the same four intervals as the CHAMP-GRACE results and thus differ in details from the full 2002 to 2010 climatologies shown in part 1.

\section{Results and discussion Diurnal components}

Figure 3 presents our comparative results for DE3 and DE2. The bottom panel illustrates the four-interval vectormean temperature amplitudes for SABER, the middle panel illustrates the same quantities for exosphere temperature derived from CHAMP and GRACE, and the top panel illustrates the CTMT exosphere temperature results based on upward HME extensions from fits to TIMED/SABER and TIMED/TIDI climatologies below $120 \mathrm{~km}$. Although there are some differences in details, the agreement in seasonal-latitudinal structures and amplitudes is sufficiently good to support the conclusion that the CHAMP-GRACE measurements reflect the vertical propagation of wave components entering the base of the thermosphere from the lower atmosphere.

The situation in Figure 4 with respect to DW2 and D0 is somewhat different. The SABER, CHAMP-GRACE, and CTMT results for DW2 are all different, and moreover, the CTMT amplitude predictions of exosphere temperature amplitude are very small, with latitudinal shape at $400 \mathrm{~km}$ considerably different than at $110 \mathrm{~km}$. Due to its short vertical wavelength, DW2 is strongly damped by molecular diffusion in the thermosphere, accounting
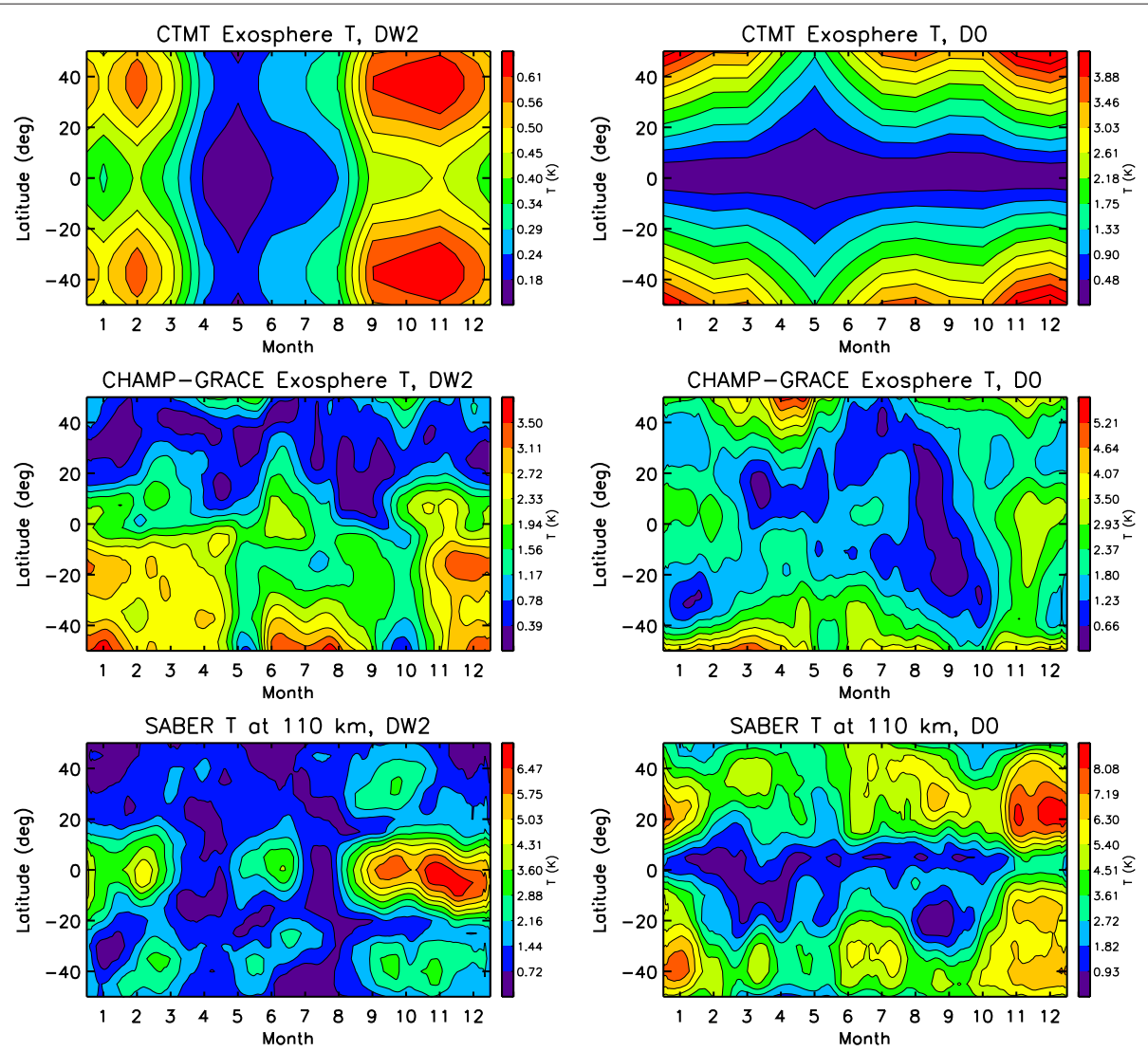

Figure 4 Same as Figure 3, except for DW2 (left) and D0 (right). 


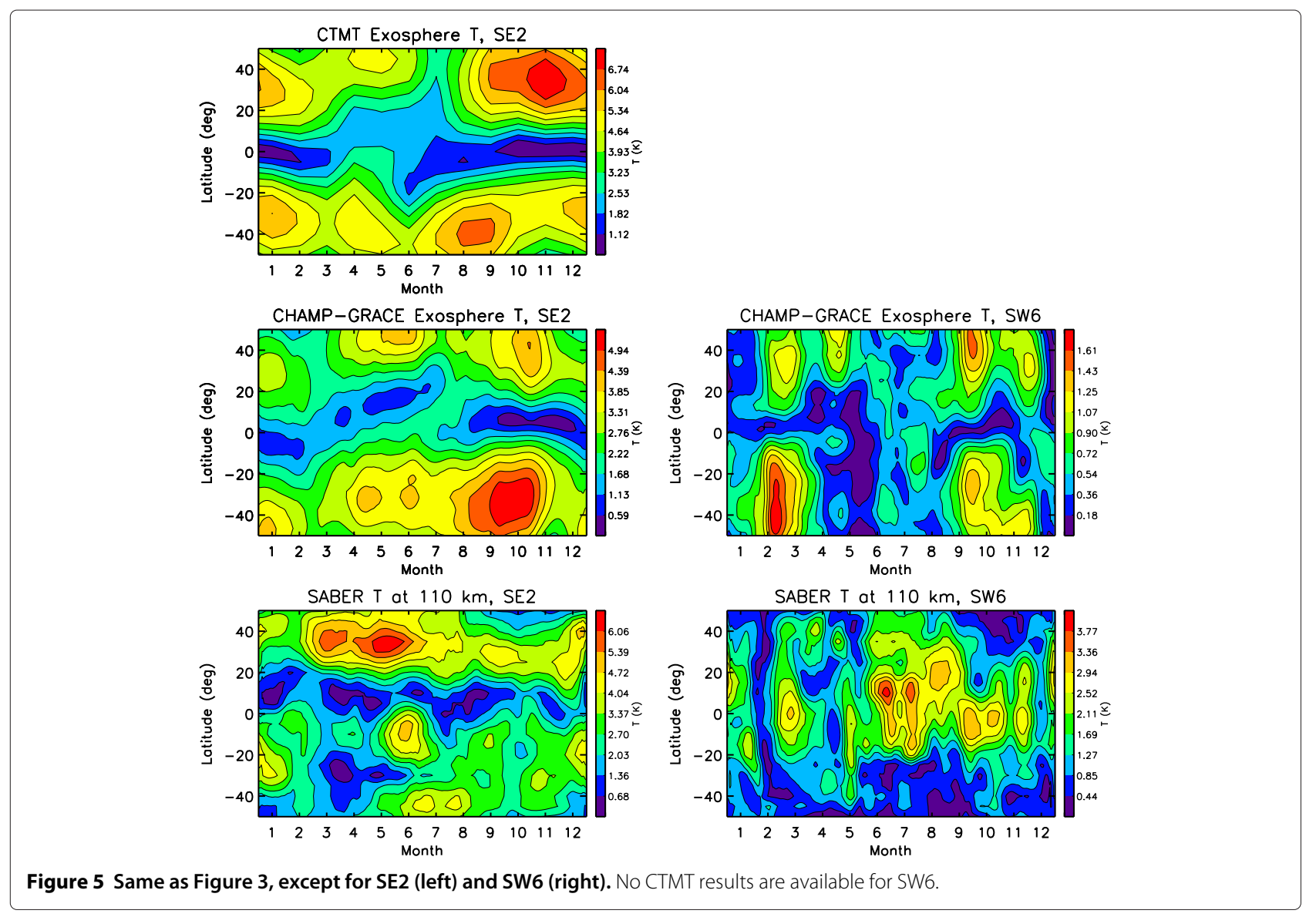

for its small amplitude at $400 \mathrm{~km}$. Moreover, because molecular dissipation renders the tidal equations for the thermosphere mathematically inseparable, coupling into trapped mode components of DW2 occurs, changing its latitudinal shape as a function of height. We conclude that DW2 in the upper thermosphere, while of the same order as DE2 and DE3 in Figure 3, is generated in situ and likely arises from the mechanisms discussed in Jones et al. (2013).

For D0, the maxima that appear around $20^{\circ}$ to $50^{\circ}$ latitude at $110 \mathrm{~km}$ appear to have broadened to beyond $50^{\circ}$ latitude in both the CHHAMP-GRACE and CTMT depictions of exosphere temperature amplitude. While the high-latitude structures appearing in the CHAMPGRACE measurements might otherwise be interpreted as a possible manifestation of polar/auroral-related perturbations, the CTMT structures, which appear at similar amplitudes, confirm that these D0 amplitudes reflect vertical propagation from the lower thermosphere. Examination of the first antisymmetric HME of D0 actually changes shape considerably with height (not shown), with maxima near $\pm 30^{\circ}$ latitude near $110 \mathrm{~km}$ and at the poles at $400 \mathrm{~km}$, consistent with the picture in Figure 4.

\section{Semidiurnal components}

Similar results for the semidiurnal tidal components are presented in Figures 5, 6 and 7. Figure 5 illustrates results for SE2 and SW6, a pair of tidal components thought to be generated by wave- 4 land-sea difference modulation of heating in the troposphere. The SE2 exosphere temperature results for CHAMP-GRACE and CTMT are in reasonable agreement in overall amplitude and seasonallatitudinal structure and thus appear to arise from vertical penetration of SE2 from $110 \mathrm{~km}$. These high-altitude amplitudes follow the first antisymmetric mode of SE2 reasonably well, which has maxima around $\pm 30^{\circ}$ latitude. However, the SE2 amplitudes are more structured at $110 \mathrm{~km}$. This is because higher-order HMEs exist at $110 \mathrm{~km}$ but become relatively more dissipated by approximately $400 \mathrm{~km}$ due to their shorter vertical wavelengths, leaving the first antisymmetric mode of SE2 dominant at higher altitudes. The differences in seasonal maxima between 110 and $400 \mathrm{~km}$ are similarly due to the presence of higher-order modes at $110 \mathrm{~km}$ which have different seasonal dependencies than the first antisymmetric mode of SE2. In contrast to D0 discussed previously, the location of maxima for this mode remains near $\pm 30^{\circ}$ latitude from 110 to $400 \mathrm{~km}$. One reason for 

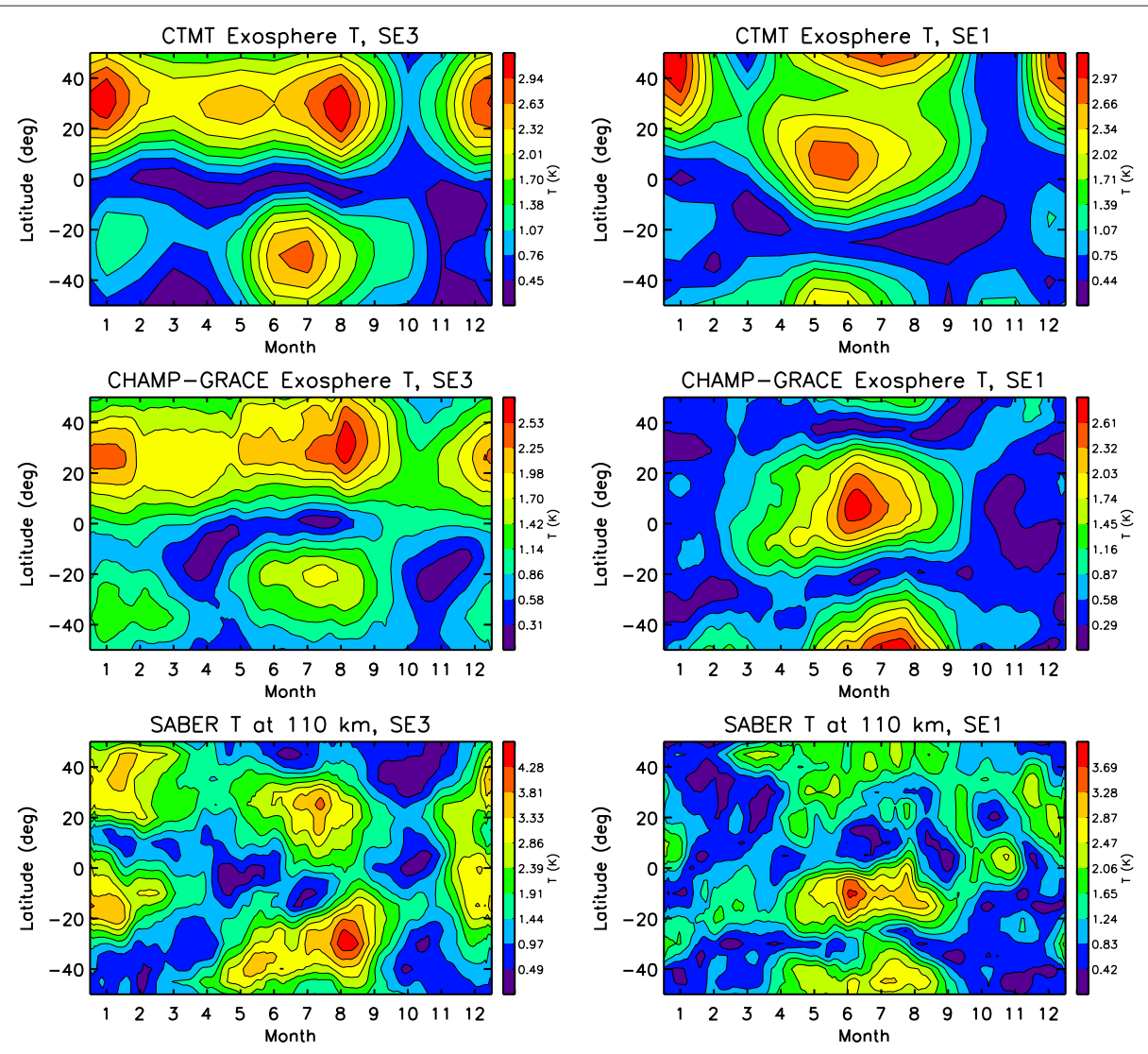

Figure 6 Same as Figure 3, except for SE3 (left) and SE1 (right).

this difference is that non-zero values are permitted at the poles for zonally symmetric $(s=0)$ oscillations, but they are not for $|s=2|$ oscillations, in order to satisfy continuity.

The right-hand column of Figure 5 provides similar results for SW6, except that CTMT results are not available for this wave component. We concluded in part 1 that SW6 at $110 \mathrm{~km}$ consists largely of its first symmetric component, but amplitudes were only of order 3 to $4 \mathrm{~K}$. From Figure 1, we see that an amplitude of $3 \mathrm{~K}$ for the first symmetric component of SW6 at $110 \mathrm{~km}$ ought to asymptote to a value of about $1.5 \mathrm{~K}$ in the upper thermosphere. This likely accounts for the absence of a clear symmetric signal for SW6 about the equator in the upper thermosphere temperatures. However, there is a well-defined but fairly small signal with minimum values at the equator. We conclude that SW6 observed in CHAMP-GRACE exosphere temperatures must arise from in situ excitation. However, there are no obvious possibilities that involve wave-wave interactions. It is possible that nonlinear interaction between SW2 and the wave-4 variation in ion drag driven by DE3 dynamo electric fields could produce SW6 and SE2 in the same way that the wave-1 component of ion drag interacts with DW1 to produce DW2 and D0 (Jones et al. 2013), but modeling studies are required to verify this conjecture.

Figure 6 shows similar results for SE1 and SE3. The correspondence in amplitude and seasonal-latitudinal structure between CHAMP-GRACE and CTMT is remarkable. The one feature of exception is the maximum predicted by CTMT near $40^{\circ}$ to $50^{\circ}$ latitude during December-January, which is absent in the CHAMP-GRACE results. It is also noteworthy that SE1 contains a relatively large contribution from the second symmetric HME component, which accounts for the maximum near the equator, while SE3 comprised mainly of the first antisymmetric HME.

Figure 7 displays results for S0 and SW4. S0 did not emerge as a major feature of the semidiurnal tide in part 1 , in part due to the relative absence of a coherent seasonallatitudinal structure that characterizes many of the other components. However, as many of the corresponding high-order HMEs dissipate with height, a more coherent pattern emerges as provided by the CTMT model in the top left panel of Figure 7. The CHAMP-GRACE results for S0 bear some gross similarity with CTMT in terms of the valley of minimum values that extends from low $\mathrm{N}$ Hemisphere latitudes in January-March, into the $\mathrm{S}$ Hemisphere during May-September, and back into the 


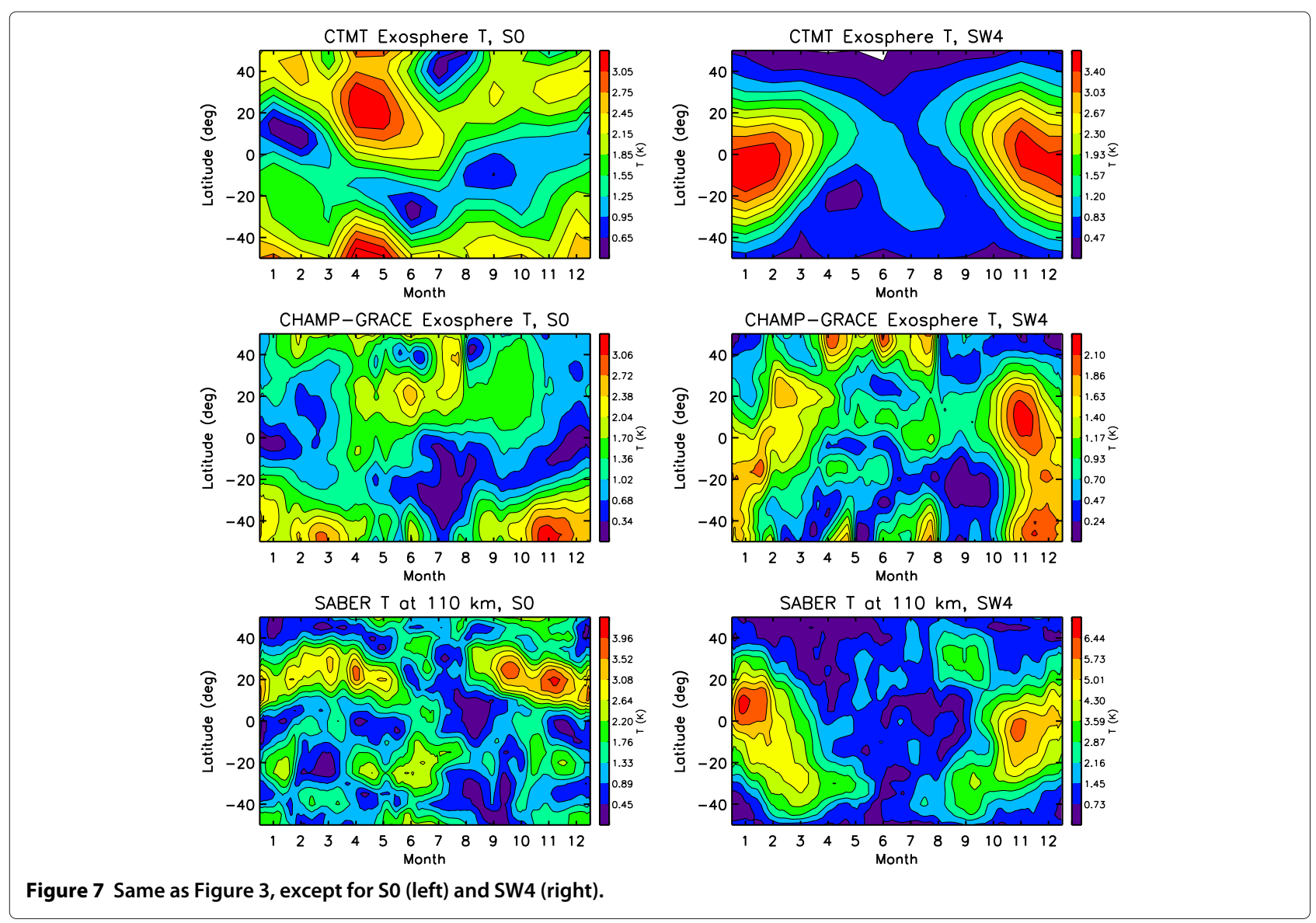

$\mathrm{N}$ Hemisphere during November-December. Amplitudes also occur at a similar level. In terms of SW4, it is clear from the SABER-CTMT comparison that the symmetric and antisymmetric components of SW4 evolve with height to shift its propensity from the $\mathrm{S}(\mathrm{N})$ to the $\mathrm{N}(\mathrm{S})$ Hemisphere as one progresses from January to April, and back again during September-December, with relatively small amplitudes during May-August. One can see similar tendencies in CHAMP-GRACE and CTMT, but a lot of higher-order structure exists in CHAMP-GRACE during May-September. Given the small amplitudes, some of this structure is noise. However, S0 and SW4 constitute another wave pair that has been shown to arise from in situ interactions in the IT (Jones et al. 2013), and this likely also contributes to the lack of better agreement between CHAMP-GRACE and CTMT.

\section{Conclusions}

In this paper, we reveal the 72-day mean diurnal and semidiurnal tidal spectra characterizing exosphere temperature between $\pm 50^{\circ}$ latitude and averaged over select periods between 2004 and 2010, i.e., average solar activity conditions. Running daily values using this 72 -day window are shown throughout the year, providing some sense of intra-seasonal variability at about half this resolution. This is made possible by exploiting the relative local time precession rates of the CHAMP and GRACE satellites. Comparisons are made with independent results in the form of the Climatological Model of Thermosphere Tides (CTMT), which uses theory-based tidal functions to extrapolate tidal fields from the 90 - to $120-\mathrm{km}$ region as determined by TIMED/SABER and TIME/TIDI measurements, to the upper thermosphere. In many cases, remarkable consistency is achieved, which serves as a validation of the technique employed to derive the tidal spectrum for the upper thermosphere and the theory that predicts the vertical propagation of tides in a diffusive thermosphere.

To summarize our results, we found strong evidence that the following waves propagate from $110 \mathrm{~km}$ to the upper thermosphere (ca. $400 \mathrm{~km}$ ) where they can be measured: DE3, DE2, D0, SE2, SE3, and SE1. DW2, S0, SW4, and SW6 tidal components are also measured in exosphere temperature. S0 and SW4 appear to consist of not only some major part that has propagated vertically from $110 \mathrm{~km}$, but also an additional component that is generated in situ. We conclude that the observed DW2 and SW6 in the thermosphere must arise from in situ excitation. 
These in situ generated tides likely arise from interactions between various waves and their interactions with the longitudinal structure of ion drag, as recently demonstrated by Jones et al. (2013). Experimental evidence for an in situ driven D0 was also found by Lieberman et al. (2013).

Results for terdiurnal tides are not shown in this paper. The non-migrating terdiurnal tides derived from CHAMP-GRACE data generally lacked well-defined seasonal-latitudinal structures and had maximum amplitudes less than $2 \mathrm{~K}$, which appears to be the level of detectability for tidal components by this method.
One impact of our results is illustrated in Figure 8. Here we show the total diurnal and semidiurnal exosphere temperature amplitudes, obtained by vectorally summing all of the derived migrating and non-migrating tidal components, at the equator and at $\pm 40^{\circ}$ latitude as a function of longitude and month. All of the longitude variability and much of the month-to-month variability illustrated here is due to the aggregate contributions of the nonmigrating tidal components, their interference with the larger migrating components. For the diurnal tide, the difference between minimum to maximum amplitudes is
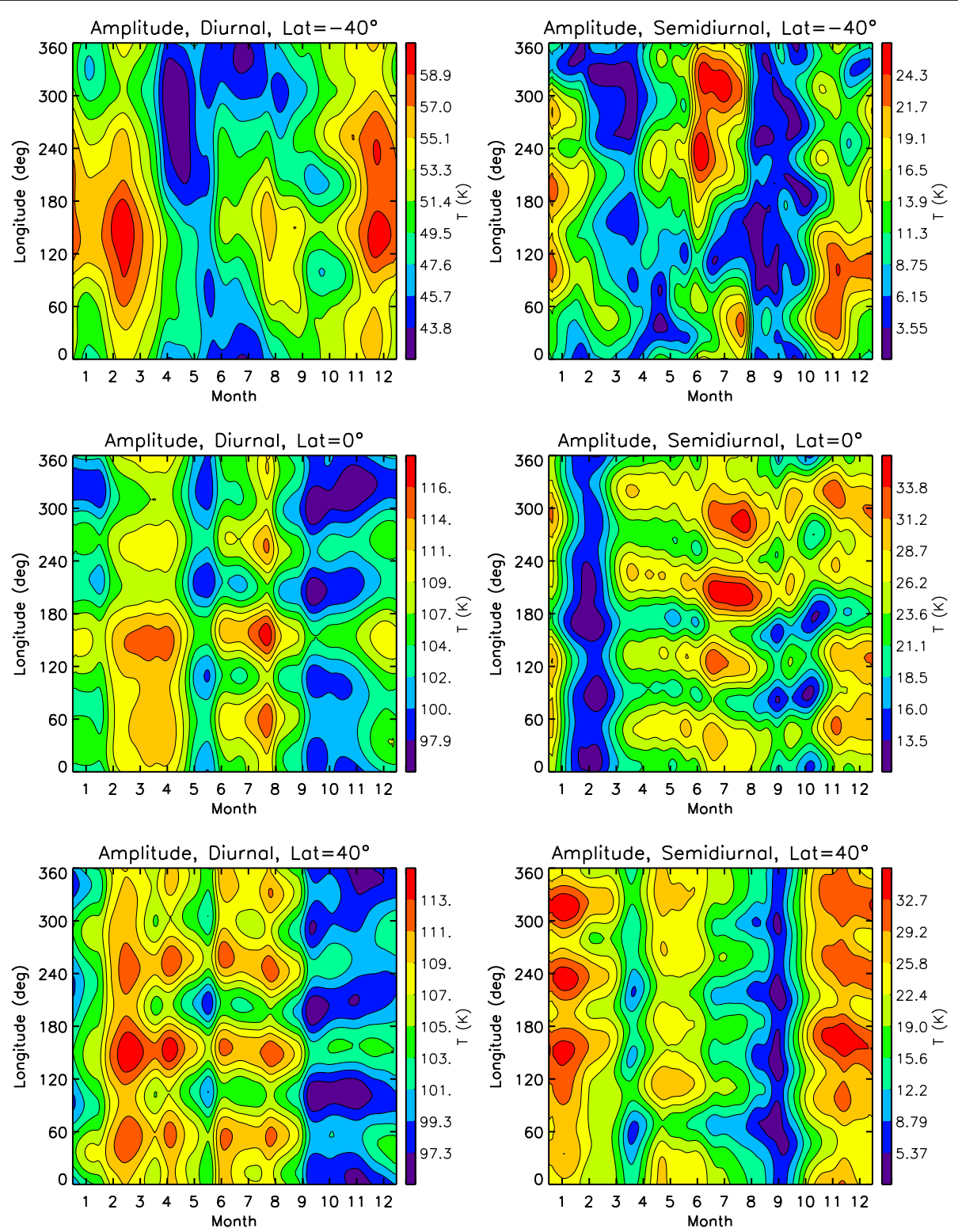

Figure 8 Diurnal and semidiurnal exosphere temperature amplitudes vs. month format. Total diurnal (left) and semidiurnal (right) exosphere temperature amplitudes in a longitude vs. month format at $-40^{\circ}$ (top), $0^{\circ}$ (middle), and $+40^{\circ}$ (bottom) latitude, obtained by vectorally superimposing the structures illustrated in Figures 3, 4, 5, 6 and 7 with the corresponding migrating diurnal and semidiurnal components. 
about $15 \mathrm{~K}$, or about $15 \%$ of the mean diurnal amplitude at $0^{\circ}$ and $40^{\circ}$ latitude and $30 \%$ at $-40^{\circ}$ latitude. For the semidiurnal tide, the variations in amplitude are much larger, $100 \%$ or more. Thus, although many of the individual tidal amplitudes may be considered small, their net effect is to impose considerable variability on the IT system. Similar levels of variability are imposed on the aggregate tidal wind fields. It is furthermore noted that all of these results pertain to the average or climatological description of the thermosphere tidal spectrum. There are in addition day-to-day changes in tides resulting from variations in forcing, changes in zonal mean propagation conditions, and planetary wave interactions that further add to tidal variability. These effects taken together impose considerable 'weather' on the IT system.

\section{Competing interests}

The authors declare that they have no competing interests.

\section{Authors' contributions}

SLB derived the CHAMP and GRACE densities from raw accelerometer data and converted the densities to exospheric temperatures. XZ processed the data and prepared the figures. JMF conceived the study, interpreted the temperature data, and wrote the paper. All authors read and approved the final manuscript.

\section{Acknowledgements}

This work was supported under Heliophysics Guest Investigator Award NNX12AJ58G from NASA to the University of Colorado.

\section{Author details \\ ${ }^{1}$ Department of Aerospace Engineering Sciences, University of Colorado, 1111 Engineering Drive, Boulder, CO 80309, USA. ${ }^{2}$ Department of Terrestrial and Planetary Geodesy, Centre National d'Etudes Spatiales 18, Avenue E. Belin, 31401 Toulouse, France.}

Received: 1 April 2014 Accepted: 16 August 2014

Published: 25 September 2014

\section{References}

Forbes JM, Hagan ME (1982) Thermospheric extensions of the classical expansion functions for semidiurnal tides. J Geophys Res 87:5253-5259

Forbes JM, Manson AH, Vincent RA, Fraser GJ, Vial F, Wand R, Avery SK, Clark RR, Johnson R, Roper R, Schminder R, Tsuda T, Kazimirovsky ES (1994) Semidiurnal tide in the 80-150 km region: an assimilative data analysis. J Atmos Terr Phys 56:1237-1250

Forbes JM, Bruinsma SL, Zhang X, Oberheide J (2009) Surface-exosphere coupling due to thermal tides. Geophys Res Lett 36:L15812. doi:10.1029/2009GL038748

Forbes JM, Zhang X, Bruinsma SL (2012) Middle and upper thermosphere density structures due to nonmigrating tides. J Geophys Res 117:A11306. doi:10.1029/2012JA018087

Geller MA (1970) An investigation of the lunar semidiurnal tide in the atmosphere. J Atmos Sci 27:202-218. http://dx.doi.org/10.1175/15200469(1970)027<0202:AIOTLS>2.0.CO;2

Hagan ME, Maute Al, Roble RG (2009) Tropospheric tidal effects on the middle and upper atmosphere. J Geophys Res 114:A01302. doi:10.1029/2008JA013637

Jones Jr M, Forbes JM, Hagan ME, Maute A (2013) Non-migrating tides in the ionosphere-thermosphere: in situ versus tropospheric sources. J Geophys Res Space Phys 118:2438-2451. doi:10.1002jgra.50257

Lieberman RS, Oberheide J, Talaat ER (2013) Nonmigrating diurnal tides observed in global thermospheric winds. J Geophys Res Space Phys 118:7384-7397. doi:10.1002/2013JA018975

Lindzen RS (1968) Vertically propagating waves in an atmosphere with Newtonian cooling inversely proportional to density. Can J Phys 46:1835-1840
Lindzen RS, Hong S-S, Forbes JM (1977) Semidiurnal Hough mode extensions into the thermosphere and their application. Memo Rep. 3442, Naval Research Laboratory, Washington, DC, p 65

Oberheide J, Forbes JM, Zhang X, Bruinsma SL (2011a) Wave-driven variability in the ionosphere-thermosphere-mesosphere system from TIMED observations: what contributes to the "wave 4"? J Geophys Res 116:A01306. doi:10.1029/2010JA015911

Oberheide J, Forbes JM, Zhang X, Bruinsma SL (2011b) Climatology of upward propagating diurnal and semidiurnal tides in the thermosphere. J Geophys Res 116:A11306. doi:10.1029/2011JA016784

Picone JM, Hedin AE, Drob DP, Aikin AC (2002) NRLMSISE-00 empirical model of the atmosphere: statistical comparisons and scientific issues. J Geophys Res 107:1468. doi:10.1029/2002JA009430

Svoboda AA, Forbes JM, Miyahara S (2005) A space-based climatology of MLT winds, temperatures and densities from UARS wind measurements. J Atmos Solar-Terr Phys 67:1533-1543

doi:10.1186/1880-5981-66-122

Cite this article as: Forbes et al.: New perspectives on thermosphere tides: 2. Penetration to the upper thermosphere. Earth, Planets and Space 2014 66:122.

\section{Submit your manuscript to a SpringerOpen ${ }^{\circ}$ journal and benefit from:}

- Convenient online submission

Rigorous peer review

- Immediate publication on acceptance

- Open access: articles freely available online

- High visibility within the field

- Retaining the copyright to your article

Submit your next manuscript at $>$ springeropen.com 